\title{
Calprotectin in serum and zonulin in serum and feces are elevated after introduction of a diet with lower carbohydrate content and higher fiber, fat and protein contents
}

\author{
BODIL OHLSSON $^{1}$, BODIL ROTH $^{1}$, EWA LARSSON $^{2}$ and PETER HÖGLUND ${ }^{3}$ \\ ${ }^{1}$ Department of Internal Medicine, Skane University Hospital, Malmo; Departments of ${ }^{2}$ Cardiothoracic Surgery \\ and ${ }^{3}$ Clinical Chemistry and Pharmacology, Lund University, Skane University Hospital, Lund, Sweden
}

Received December 21, 2016; Accepted February 1, 2017

DOI: $10.3892 / b r .2017 .865$

\begin{abstract}
Calprotectin is a marker of inflammation and zonulin is a marker of intestinal permeability. Diets with lower carbohydrate content and higher contents of fat, fiber and protein, e.g., Okinawan-based diet, are considered to reduce inflammation and intestinal permeability. The aim of the present study was to evaluate calprotectin and zonulin levels in serum and feces after intervention with an Okinawan-based Nordic diet. Thirty patients (17 women) with type 2 diabetes, mean age $57.5 \pm 8.2$ years, BMI $29.9 \pm 4.1 \mathrm{~kg} / \mathrm{m}^{2}$, were served the diet during 12 weeks, and were followed for another 16 weeks. Anthropometric and metabolic parameters were registered. Fasting levels of calprotectin and zonulin in serum and feces, and hormones in plasma, were measured by Luminex or ELISA before study start and after 12 and 28 weeks. Calprotectin in serum tended to be increased $(\mathrm{P}=0.074)$ after 12 weeks. Zonulin in serum and feces were elevated after 12 weeks $(\mathrm{P}=0.019$ vs. $\mathrm{P}<0.001)$, and remained elevated in serum after 28 weeks $(P=0.014)$. In contrast to baseline, there was a correlation between calprotectin and zonulin in serum and feces after dietary intervention $(\mathrm{P}=0.025$ vs. $\mathrm{P}=0.079)$. Energy percentage of protein in breakfast correlated with serum calprotectin $(\mathrm{P}=0.008)$ and tended to correlate with serum zonulin $(\mathrm{P}=0.059)$. Calprotectin in serum tended to be elevated, and zonulin in serum and feces are elevated after introduction of an Okinawan-based Nordic diet. These biomarkers correlate with energy percentage of protein.
\end{abstract}

\section{Introduction}

Calprotectin is a heterodimer of the subunits S100A8 (calgranulin A, MRP8) and S100A9 (calgranulin B, MRP14), which

Correspondence to: Dr Bodil Ohlsson, Department of Internal Medicine, Skane University Hospital, Inga Marie Nilsson Street 32, S-205 02 Malmo, Sweden

E-mail: bodil.ohlsson@med.lu.se

Key words: calprotectin, fat content, fiber content, low carbohydrate content, Okinawan-based Nordic diet, protein content, zonulin are low-molecular-weight members of a subfamily of S100 calcium-binding proteins called calgranulins (1). The complex is mainly expressed in cells of the myeloid lineage, but is also expressed in macrophages, osteoclasts, keratinocytes, fibroblasts, and microvascular endothelial cells $(2,3)$. Apart from functions related to anti-infectious host defence mechanisms, calprotectin is involved in kinase activities, cytoskeletal rearrangement, and cell differentiation and migration (3). Calprotectin has emerged as a mediator of chronic inflammation (4-6), since calprotectin substantially contributes to the recruitment of monocytes to an inflammatory site (7).

The paracellular intestinal permeability is controlled by at least four intercellular junctions that regulate the space between epithelial cells and are regularized by a set of over 50 proteins. One of the best well-described junctions are tight junctions (8). Zonulin is the eukaryotic counterpart of the Vibrio cholerae zonula occludens toxin (9). Human zonulin (47-kDa protein), also known as prehaptoglobin-2, which binds to epidermal growth factor receptor via protease-activated receptor 2 activation. This complex initiates a signalling pathway that results in phosphorylation of zonula occludens proteins and leads to small intestine tight junction disassembling (10). Zonulin is the only measurable blood protein that reflects the intestinal permeability, and increased levels are considered to be a marker of impaired intestinal barrier $(11,12)$. The secretion of zonulin is triggered by gluten and bacteria (12), and as zonulin increases the intestinal permeability, the ability for the washout of bacterial colonization in the gut is improved (13). In contrast, haptoglobin is a protein secreted mainly by hepatocytes with immune-modulating properties, showing anti-inflammatory effects (14). Thus, the presence of zonulin does not only reflect intestinal permeability, but may also reflect a reaction secondary to inflammation.

Obesity and type 2 diabetes are conditions characterized by chronic inflammation and increased intestinal permeability (15). Modern Western food is considered to be pro-inflammatory and is associated with hyperinsulinemia, increased intestinal permeability, and micro-inflammation $(16,17)$. High intake of plant-derived foods, and a lower intake of red meat, meat products, sweets, salt, high-fat dairy, and refined grains, are considered to be important features of healthy diets (18). These features are found in 
an Okinawan-based Nordic diet, developed to represent a healthier diet with tastes and food suitable for the Nordic population. Since many systemic diseases are considered to depend on chronic inflammation and interactions with intestinal permeability and microbiota, it is of great importance to find a healthy diet with optimal effects on the gastrointestinal tract (17).

Our hypothesis was that the Okinawan-based Nordic diet with lower carbohydrate content and energy intake, and higher fat, fiber, and protein contents, influences the secretion of calprotectin and zonulin in type 2 diabetes. The diet was given to patients with type 2 diabetes during 12 weeks, with a follow-up for another 16 weeks. Blood and feces samples and clinical examinations were taken before study start, and after 12 and 28 weeks. The complete description of the present study methodology and results on anthropometric and metabolic parameters have been presented in a previous report (18). The primary objective of the present study was to measure serum and feces levels of calprotectin and zonulin. The secondary objectives were to determine correlations between these factors and food components, anthropometry, hormonal levels, other inflammatory biomarkers, and metabolic responses.

\section{Materials and methods}

The subjects were treated according to the Helsinki declaration and the study was approved by the Regional Ethics Review Board at Lund University (2014/460). All subjects gave their written, informed consent before entering the study which was monitored by an external monitor and registered at ClinicalTrials.gov data base (NCT02405806).

Study population. Patients with type 2 diabetes, independently of body mass index (BMI) or anti-diabetic treatment regimen, aged between 18-70 years, were recruited among diabetes patients at a primary health care center in the southernmost district of Sweden. Patients were to have both parents inborn in Scandinavia, to avoid possible influence of ethnicity on the study results. Overall exclusion criteria were severe food allergy, prior major gastrointestinal surgery, severe diseases including renal and liver insufficiency, type 1 diabetes, and alcohol and drug abuse. Participants were initially informed through a letter sent by mail of the project design and purpose. One week later, all the patients were contacted by phone.

Study design. A prospective interventional study with an Okinawan-based Nordic diet was conducted during 12 weeks, followed by a clinical follow-up after 16 weeks with unrestricted diets, at Skåne University Hospital, Malmö, Sweden. All tests were performed under standardized conditions. The study data consisting of blood and feces sampling; assessments of anthropometric data; and completion of questionnaires and protocols was obtained at three separate visits: i) At study start before introduction of the Okinawan diet; ii) after 12 weeks on the Okinawan diet; and iii) after 16 weeks on unrestricted diet (Fig. 1). Food for preparation of lunch and dinner was delivered to the subjects three times a week. They were allowed to visit a restaurant or to ingest another meal at most once weekly. The participants had to prepare their own breakfast and were encouraged to maintain their regular physical activity habits.
Physical examination included abdominal, cardiopulmonary, and neurological examinations as well as measurement of blood pressure, pulse, respiratory rate, weight, height, waist circumference, and assessment of BMI. Feces was collected at home by the patient before each hospital visit. Blood samples were collected through an intravenous catheter after a $10 \mathrm{~h}$ fast. Metabolic parameters were analyzed at once, and plasma and serum were harvested and stored at $-80^{\circ} \mathrm{C}$ until analyzed for hormonal and inflammatory concentrations. The study started on 2 February 2015 and ended on 18 September 2015.

Questionnaires. A study questionnaire about socio-economic factors, family history, smoking and alcohol habits, physical activity, health history, and drug treatment was completed at all three time-points. In addition, the subjects reported about compliance to the diet and life events since last control.

Diets. The diet is based on the traditional Okinawan diet (19), but modified to suit tastes and food components suitable for the Nordic population. The diet and the study protocol is described in detail in a previous publication (18). Briefly, the meal composition is consistent with moderately low carbohydrate content (20). The contents of fiber, fat, and protein are increased, which lead to a bigger meal demanding more mastication and prolonged meal intake (21). The food is based on traditional Nordic raws, e.g., whole grains, vegetables, leguminous, root crops, fat fish, birds, fruits, berries, and nuts, with minimal industrial processing. The amount of dairy products, read meat, and processed meat was limited, as well as sugar and white flour to have a diet with low glycemic index (GI). The gluten content was decreased since almost no wheat meal was used, and the participants only were allowed to ingest 2 slices of bread each day and no cakes were given. The fiber content in their breakfast was increased non-significantly during dietary intervention from $3.3 \pm 1.7$ to $4.0 \pm 1.4 \mathrm{E} \%$ ( $\mathrm{P}=0.131)$. The diet has a good nutritional supply including a mean calorie intake around 1,900 kcal/day, which is slightly lower compared with a traditional diet. The participants were allowed to eat breakfast, lunch, and dinner, and two snacks between meals consisting of a variety of fruits, berries, and seeds. Organic food items were preferred whenever possible. At the occurrence of cravings, the subjects were instructed to eat a third snack (e.g., carrots, boiled eggs, mackerel in tomato sauce, or cottage cheese with berries) to avoid eating fast carbohydrates. Each meal started with ingestion of raw vegetables or green salad; $100 \mathrm{~g}$ at breakfast and $150 \mathrm{~g}$ at lunch and dinner, respectively. Nutrition information is given in Table I.

Two breakfast alternatives were suggested, depending on their ordinary breakfast ingested and consisted of either porridge or fermented milk, and subjects had to buy the ingredients themselves. Those who did not get any fermented milk product to breakfast ingested any form of fermented milk as a snack, so all subjects ingested Lactobacillus acidophilus, Bifidobacterium lactis, and Streptococcus thermophiles every day. The other meals were planned at the kitchen of Igelösa Life Science Lab (Lund University) and delivered to the subjects regularly free of charge, along with recipes and instructions on how to cook the food. No other changes in diet or supplements were allowed to be introduced during the study period. Maximal intake of alcoholic beverages was set to $30 \mathrm{~g}$ ethanol/week. 
Table I. Nutrition composition and daily mean intake of energy, nutrients, and food components of the modified Okinawan-based Nordic diet, compared with Nordic Nutrition Recommendations 2012.

\begin{tabular}{|c|c|c|c|c|}
\hline Nutritional value & Unit & Calculated value & $\mathrm{E} \%$ & Recommended (NNR 2012) \\
\hline Total Energy & kcal & 1866 & & \\
\hline Energy (excluding beverages) & kcal & 1629 & & \\
\hline Carbohydrate & g & 168.4 & 42 & $45-60 \mathrm{E} \%$ \\
\hline Sucrose & $\mathrm{g}$ & 23.5 & 6 & $<10 \mathrm{E} \%$ \\
\hline Dietary fibre & $\mathrm{g}$ & 35.9 & & $25-35 \mathrm{~g}$ \\
\hline Fat & $\mathrm{g}$ & 63.9 & 35 & $25-40 \mathrm{E} \%$ \\
\hline Saturated fatty acids & $\mathrm{g}$ & 18.7 & 10 & $<10 \mathrm{E} \%$ \\
\hline Polyunsaturated fatty acids & $\mathrm{g}$ & 14.9 & 8 & $5-10 \mathrm{E} \%$ \\
\hline Mono-unsaturated fatty acids & $\mathrm{g}$ & 17.8 & 10 & $10-20 \mathrm{E} \%$ \\
\hline Protein & $\mathrm{g}$ & 95.0 & 23 & $10-20 \mathrm{E} \%$ \\
\hline
\end{tabular}

Daily mean intake of energy, nutrients, and food components $(n=30)$ of the modified Okinawan-based Nordic diet, compared with Nordic Nutrition Recommendations (NNR) 2012 (20).

Table II. Coefficient of variations for controls in Luminex and ELISA analyses.

\begin{tabular}{|c|c|c|c|c|c|}
\hline Biomarker & $\begin{array}{c}\text { Low control } \\
\text { Inter-assay } \mathrm{CV}, \% \text { (n) }\end{array}$ & $\begin{array}{c}\text { High control } \\
\text { Inter-assay } \mathrm{CV}, \% \text { (n) }\end{array}$ & $\begin{array}{c}\text { Intra-assay } \\
\mathrm{CV}^{\mathrm{a}} \%\end{array}$ & $\begin{array}{c}\text { Low control } \\
\text { Intra-assay } \mathrm{CV}, \% \text { (n) }\end{array}$ & $\begin{array}{c}\text { High control } \\
\text { Intra-assay } \mathrm{CV}, \%(\mathrm{n})\end{array}$ \\
\hline Serum calprotectin & $5.5(10)$ & $7.0(10)$ & & $3.7(80)$ & $7.9(80)$ \\
\hline Feces calprotectin & $4.4(12)$ & $8.9(12)$ & & $5.6(20)$ & $4.4(12)$ \\
\hline Serum zonulin & $13.3(14)$ & $13.6(14)$ & & $3.4(40)$ & $6.0(40)$ \\
\hline Feces zonulin & $12.7(16)$ & $13.9(16)$ & & $3.2(24)$ & $5.9(24)$ \\
\hline PAI-1 & $7.4(5)$ & $12.8(4)$ & 5 & & \\
\hline Resistin & $4.3(8)$ & $10.5(8)$ & 3 & & \\
\hline Visfatin & $29.0(8)$ & $15.5(8)$ & 4 & & \\
\hline
\end{tabular}

antra-assay coefficient of variation (CV) \% is according to Bio-Rad Laboratories (Richmond, CA, USA). CV for calprotectin and zonulin are from the manufacturer Immundiagnostik AG (Bensheim, Germany).

Blood and feces sampling and chemistry analyses. All samples consisted of whole blood drained into ethylenediaminetetra-acetic acid glass tubes (BD Microtainer; BD Biosciences, Franklin Lakes, NJ, USA) or serum separation tubes with coagulation activator and gel (BD Biosciences). Blood was immediately cooled, centrifuged at 3,000 x g for $10 \mathrm{~min}$, and plasma and serum was stored in $-80^{\circ} \mathrm{C}$ until analyzed. C-peptide and insulin in serum; albumin, C-reactive peptide (CRP), glucose, haptoglobin, liver function tests, cholesterol, high-density lipoprotein (HDL), low-density lipoprotein (LDL), and triglycerides in plasma; and leukocytes, thrombocytes, and hemoglobin A1c (HbA1c) in blood were analyzed by standard methods at the Department of Clinical Chemistry at Skåne University Hospital, Malmö, Sweden. Homeostasis model assessment for insulin resistance (HOMA2-IR) was calculated using the HOMA2 calculator version 2.2.3 (22).

Feces were collected at home in sterile tubes (80.9924.014 polypropylene; Sarstedt AG \& Co., Nümbrecht, Germany) at inclusion, at week 12 and 28. The samples were collected 1-2 days before the scheduled visit, and stored in the deep-freezer at home until delivery to the hospital. Samples were stored at $-80^{\circ} \mathrm{C}$ (stabilisation guarantee for 1 year from the manufacturer) until further analyzed within 6 weeks.

Calprotectin. The calprotectin concentration was determined by using an enzyme-linked immunosorbent assay (ELISA) kit (Immundiagnostik AG, Bensheim, Germany, batch no. K 6935-141117 for all serum samples and K 6927-150120 for all feces samples). The assay used the two-site sandwich technique in which the intensity of the color was directly related to the calprotectin concentration in the sample. Samples were read at $450 \mathrm{~nm}$, and the 4-parameter algorithm was used to form the standard curve and to calculate data. All tests were carried out in duplicate in a blinded manner within 6 weeks after sampling with the same batch numbers. Calprotectin concentration in serum is presented in $\mathrm{ng} / \mathrm{ml}$ and reference value in healthy volunteers is $<3,000 \mathrm{ng} / \mathrm{ml}$, according to the manufacturer. Concentration in feces is presented as $\mathrm{mg} / \mathrm{kg}$. Mean value in healthy volunteers is $25 \mathrm{mg} / \mathrm{kg}$ and $>50 \mathrm{mg} / \mathrm{kg}$ is considered a positive value (23). Inter-assay and intra-assay coefficients of variance (CV) for controls are shown in Table II. 
Zonulin. The zonulin concentration was determined by using an ELISA kit (Immundiagnostik AG, batch no. K5601-150309 for all serum samples and K5600-150220 for all feces samples). The assay used the competitive binding technique. Biotinylated zonulin tracer was added to the samples, standards, and positive and negative controls as a competitor to the sample's own zonulin. The intensity of the color was inversely proportional to the zonulin concentration in the sample. Samples were read at $450 \mathrm{~nm}$, and the 4-parameter algorithm was used to form the standard curve and to calculate data. All tests were carried out in duplicate in a blinded manner within 6 weeks after sampling with the same batch numbers. Zonulin concentration is presented in $\mathrm{ng} / \mathrm{ml}$. Based on the manufacturer's studies of apparently healthy persons $(n=40)$, a mean value of $34 \pm 14 \mathrm{ng} / \mathrm{ml}$ was estimated in serum and a mean value of $61 \pm 46 \mathrm{ng} / \mathrm{ml}$ was estimated in feces. Inter-assay and intra-assay CV for controls are shown in Table II.

Hormonal analyses. The Luminex analyses were performed within 9 months. Human diabetes 10-plex panel (Bio-Plex Pro $^{\text {TM }}$ Human Diabetes Immunoassay control nos. 5029560-1 and 5040782; Bio-Rad Laboratories, Richmond, CA, USA) was performed on the Luminex-200 (Luminex xMAP; Bio-Rad Laboratories) and data were analyzed using Bio-Plex Manager software 6.0 (Bio-Rad Laboratories). Hormones (pg/ml) measured were ghrelin, glucagon, glucagon-like peptide-1 (GLP-1), glucose-dependent insulinotropic polypeptide (GIP), leptin, serpin plasminogen activator inhibitor-1 (PAI-1), resistin, and visfatin, also called nicotinamide phosphoribosyltransferase (NAMPT) or pre-B-cell colony-enhancing factor.

Analyses were performed blinded according to the manufacturers' instructions. Briefly, samples were diluted 1:4 and incubated with magnetic beads coupled to specific capture antibodies. After a series of washes in a magnetic wash station (Bio-Plex Handled magnetic washer, 171020100; Bio-Rad Laboratories), biotinylated detection antibodies were added to form a sandwich complex. The final detection complex was created with the addition of streptavidin-phycoerythrin conjugate. Absolute concentrations were measured from standards provided with the kit. Each run included controls with high and low concentrations for each biomarker, and a blank sample. All samples were analyzed in duplicate and the concentration of hormones bound to each bead was proportional to the median fluorescence intensity of reporter signal. Standard curves were calculated with type 5-parameter nonlinear logistic regression. Inter-assay and intra-assay $\mathrm{CV}$ for controls are presented in Table II.

Cholecystokinin (CCK) and polypeptide YY (PYY) were measured by a commercial competitive inhibition ELISA (CEB802 $\mathrm{Hu}$ and CEB067 $\mathrm{Hu}$, respectively), (Cloud-Clone Corp, Houston, TX, USA), kit according to the manufacturer's instructions. Intra-assay and inter-assay are CV\% $<10 \%$ and $<12 \%$, respectively, for both CCK and PYY.

Statistical methods. Three hypotheses were raised: i) An Okinawan-based Nordic diet affects the release of calprotectin and zonulin; ii) there is a correlation between concentrations of calprotectin and zonulin in feces and serum; and iii) there are correlations between calprotectin and zonulin levels and food components, hormonal secretion, other inflammatory biomarkers, metabolic responses, and changes in weight, BMI, and waist circumference.

A power analysis was performed based on a previous unpublished pilot study, and we determined that nine subjects was required to demonstrate clinically significant differences in metabolic parameters with $80 \%$ power at $5 \%$ significance level. The variable demanding most persons to be able to discover was, what we expected, the diastolic blood pressure. We determined that we needed 18 persons to demonstrate clinically significant differences in diastolic pressure with $80 \%$ power at $5 \%$ significance level. To be able to compensate for disappearance, we planned to recruit 25-35 persons. Two patients interrupted the study at 6 weeks on diet, which time point was considered as the end of the intervention, and data collected at that time point was calculated together with data from patients with 12 weeks of intervention. Normal weight was defined as BMI $<25 \mathrm{~kg} / \mathrm{m}^{2}$ and obese as BMI $\geq 30 \mathrm{~kg} / \mathrm{m}^{2}$ (24).

We tested the hypotheses with linear mixed effect models to analyze continuous variables, with random intercept and unstructured covariance's for repeated measures within a patient, with visits as nominal fixed effect, using baseline as reference. We assumed that missing observations were unrelated to the observed value, i.e., missing at random. In these analyses, predicted mean values and their $95 \%$ confidence limits are presented, together with estimates of changes from baseline and 95\% confidence limits and P-values for the changes. Descriptive statistics are presented as means and standard deviations for continuous variables and as counts or frequencies for categorical variables. Spearman's correlation test was used for correlations. As the study diet except breakfast was standardized, and the vast majority of patients kept this breakfast during follow-up, we calculated the food composition in the breakfasts in correlation to calprotectin, zonulin, and other inflammatory biomarker levels at baseline, week 12 and 28. Mann-Whitney U test was used to describe differences between subgroups. Statistics were done using MATLAB R2015a (Mathworks Inc.). P $\leq 0.05$ was considered to indicate a statistically significant difference.

\section{Results}

Patient characteristics. In total, 45 patients were randomly selected after consideration of inclusion and exclusion criteria. In all, 30 patients (17 women), mean age $57.5 \pm 8.2$ years, were included from these 45 invited patients (Fig. 1). Reasons for not inclusion were: Unwillingness to participate $(n=11)$, late autoimmune diabetes in adult $(\mathrm{n}=1)$, a history of gastric by-pass surgery $(n=1)$, pregnancy $(n=1)$, or already on a diet $(n=1)$. The disease duration of type 2 diabetes was $10.4 \pm 7.6$ years. The treatment was metformin $(40 \%)$, metformin in combination with insulin (27\%), insulin solely (13\%), metformin in combination with sulfonylurea $(7 \%)$, diet solely $(7 \%)$, sulfonylurea $(3 \%)$, or dipeptidyl peptidase-4 $(3 \%)$. The most common secondary complication to the disease was autonomic neuropathy and/or peripheral neuropathy (30\%), retinopathy (27\%), and nephropathy and macroangiopathy (17\% in both). Only one patient had a verified gastroparesis.

The majority, $67 \%$, were employed, whereas $17 \%$ were retired. The rest of the patients were on sick leave or unemployed. Twenty of the patients were married or cohabitant, whereas six 
Recruitment of patients with type 2 diabetes at a primary health care center

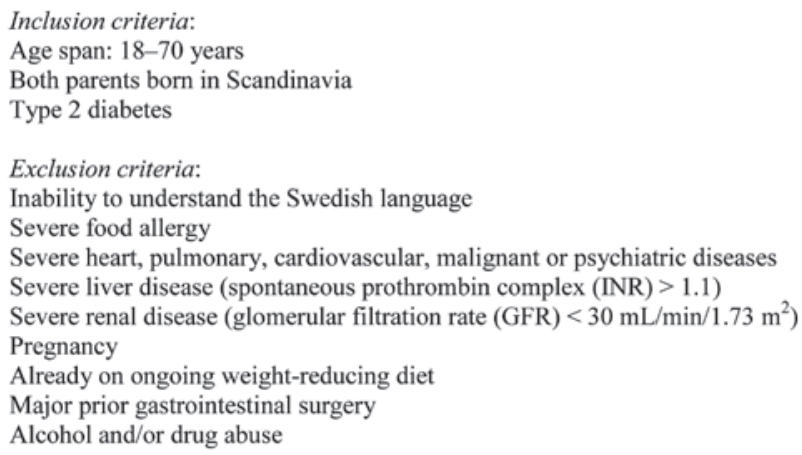

45 available patients contacted by mail and phone

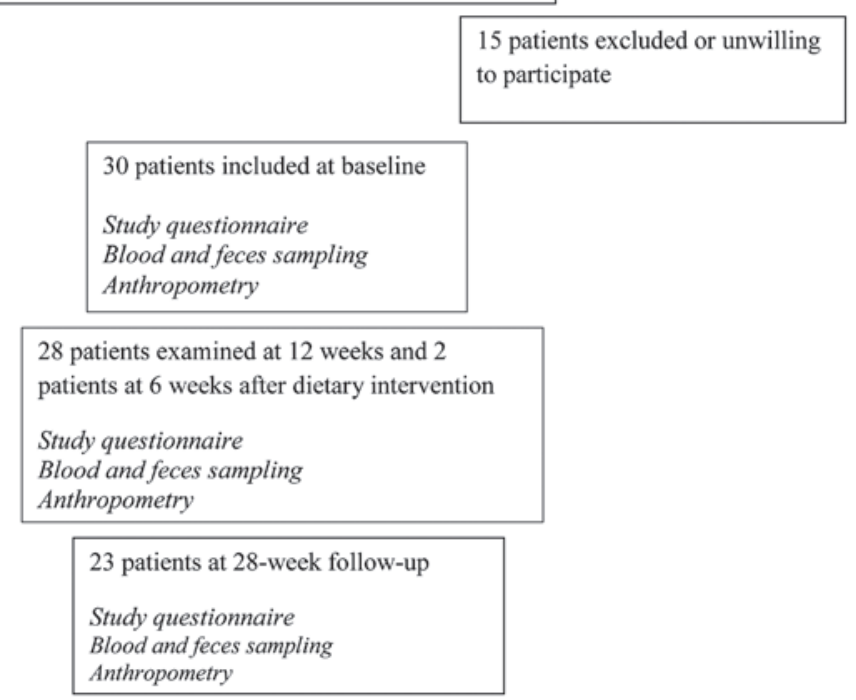

Figure 1. Flow-chart of patient recruitment and study design. Two patients interrupted the study at 6 weeks on diet, and data collected at that time-point was calculated together with data from patients with 12 weeks of intervention.

were divorced or widow/widower, and four were living alone. Smoking and snuff using both occurred in $23 \%$ of the patients. Half of the patients drank alcoholic beverages once a month or less, 27\% drank alcohol 2-4 times a month, 13\% drank alcohol 2-3 times a week, and $10 \%$ were teetotalers. A moderately physical exercise during leisure time was most common (53\%), followed by a moderately regular exercise $(27 \%)$, regular exercise and training (13\%), and sedentary leisure time (7\%). Five patients (13\%) used probiotics regularly.

During the interventional period, one subject was operated on for hernia and one for hip arthroplasty. During the same time, some subjects had infections in the airways $(n=4)$, teeth $(n=1)$, and urinary tract $(n=1)$, which did not affect the concentrations of any inflammatory biomarkers (data not shown). During the follow-up, infections in the airways $(n=1)$ and teeth $(n=1)$ were seen. One subject had severe exacerbation from his psoriasis throughout the study. Both subjects who suffered and were treated from severe depression interrupted their participation at week 12 .

At follow-up, almost all patients had kept their regular meal order with the same intake of breakfast as during the intervention, and with an increased intake of vegetables and legumes. The composition of lunch and dinner was partly withheld.

Calprotectin. Calprotectin levels in serum were within normal ranges although a tendency to increased levels during the dietary intervention $(\mathrm{P}=0.074)$, which returned to basal values at week 28 . Feces calprotectin values were higher than the normal reference level $(<50 \mathrm{mg} / \mathrm{kg})$ and were decreased non-significantly throughout the study (Table III). Calprotectin levels in serum and feces did not correlate when correlated at the three time points measured (data not shown). Levels did not differ between gender, normal-weighted and over-weighted subjects, or non-obese and obese subjects (data not shown).

Zonulin. Compared with the mean value of $34 \pm 14 \mathrm{ng} / \mathrm{ml}$ in healthy volunteers, zonulin values in serum were elevated throughout the study (Table III). On the contrary, the feces levels were only higher than expected $(61 \pm 46 \mathrm{ng} / \mathrm{ml})$ after 12 weeks of dietary intervention, whereas baseline levels and follow-up levels were within normal ranges (Table III). Zonulin 
Table III. Inflammatory biomarkers.

\begin{tabular}{|c|c|c|c|c|c|c|c|}
\hline \multirow[b]{2}{*}{ Variable } & \multirow[b]{2}{*}{ Mean } & \multicolumn{2}{|c|}{$95 \% \mathrm{CI}$} & \multirow[b]{2}{*}{ Mean change } & \multicolumn{2}{|c|}{$95 \% \mathrm{CI}$} & \multirow[b]{2}{*}{ P-value } \\
\hline & & Lower & Upper & & Lower & Upper & \\
\hline \multicolumn{8}{|c|}{ Serum calprotectin (ng/ml) } \\
\hline Baseline & 1816 & 1502 & 2130 & & & & \\
\hline Week 12 & 2187 & 1785 & 2588 & 370.8 & -37.3 & 778.9 & 0.074 \\
\hline Week 28 & 1460 & 1003 & 1916 & -356.2 & -818.1 & 105.7 & 0.129 \\
\hline \multicolumn{8}{|c|}{ Feces calprotectin (mg/kg) } \\
\hline Baseline & 141.1 & 71.8 & 210.5 & & & & \\
\hline Week 12 & 137.7 & 60.8 & 214.5 & -3.5 & -48.4 & 41.4 & 0.878 \\
\hline Week 28 & 131.1 & 37.0 & 225.2 & -10.0 & -80.6 & 60.5 & 0.778 \\
\hline \multicolumn{8}{|c|}{ Serum zonulin (ng/ml) } \\
\hline Baseline & 79.9 & 71.3 & 88.5 & & & & \\
\hline Week 12 & 92.9 & 83.5 & 102.3 & 12.9 & 2.2 & 23.7 & 0.019 \\
\hline Week 28 & 95.1 & 84.2 & 105.9 & 15.2 & 3.2 & 27.2 & 0.014 \\
\hline \multicolumn{8}{|c|}{ Feces zonulin (ng/ml) } \\
\hline Baseline & 49.4 & 38.3 & 60.4 & & & & \\
\hline Week 12 & 92.3 & 71.3 & 113.2 & 42.9 & 21.0 & 64.8 & $<0.001$ \\
\hline Week 28 & 53.5 & 40.4 & 66.6 & 4.2 & -10.4 & 18.7 & 0.572 \\
\hline \multicolumn{8}{|c|}{ Albumin (g/l) } \\
\hline Baseline & 39.1 & 38.0 & 40.2 & & & & \\
\hline Week 12 & 38.8 & 37.6 & 40.0 & -0.3 & -1.3 & 0.7 & 0.560 \\
\hline Week 28 & 39.4 & 38.2 & 40.7 & 0.3 & -0.7 & 1.4 & 0.523 \\
\hline \multicolumn{8}{|c|}{ C-reactive protein $(\mathrm{mg} / \mathrm{l})$} \\
\hline Baseline & 3.7 & 1.8 & 5.6 & & & & \\
\hline Week 12 & 2.9 & 1.0 & 4.8 & -0.8 & -3.5 & 1.9 & 0.569 \\
\hline Week 28 & 1.7 & -0.5 & 3.9 & -2.0 & -4.9 & 0.9 & 0.173 \\
\hline \multicolumn{8}{|c|}{ Haptoglobin (g/l) } \\
\hline Baseline & 1.4 & 1.3 & 1.6 & & & & \\
\hline Week 12 & 1.5 & 1.3 & 1.7 & 0.1 & -0.1 & 0.2 & 0.605 \\
\hline Week 28 & 1.3 & 1.2 & 1.5 & -0.1 & -0.2 & -0.0 & 0.009 \\
\hline \multicolumn{8}{|c|}{ Leucocytes (x109/1) } \\
\hline Baseline & 6.7 & 6.1 & 7.3 & & & & \\
\hline Week 12 & 6.6 & 5.8 & 7.3 & -0.1 & -0.7 & 0.4 & 0.660 \\
\hline Week 28 & 6.5 & 5.8 & 7.2 & -0.2 & -0.7 & 0.3 & 0.498 \\
\hline \multicolumn{8}{|c|}{ Thrombocytes (x109/1) } \\
\hline Baseline & 264.4 & 242.0 & 286.9 & & & & \\
\hline Week 12 & 258.3 & 233.2 & 283.3 & -6.2 & -25.1 & 12.8 & 0.520 \\
\hline Week 28 & 237.3 & 211.9 & 262.7 & -27.1 & -46.6 & -7.6 & 0.007 \\
\hline
\end{tabular}

The mean values and mean changes and 95\% confidence interval (CI) with lower and higher limits are presented for fasting levels of calprotectin and zonulin (serum and feces), albumin, C-reactive protein, and haptoglobin (plasma), and leucocytes and thrombocytes (whole blood), at inclusion (baseline), 12 weeks after dietary intervention, and 16 weeks after the end of dietary intervention (week 28). Linear mixed model. $\mathrm{P} \leq 0.05$ was considered statistically significant.

in both serum and feces was increased during the dietary intervention, and this increase persisted in serum during the follow-up (Table III). There was no correlation between zonulin levels in serum and feces at the three time-points measured (data not shown). Use of probiotics tended to lower zonulin levels in feces at baseline $(28.5 \pm 8.8 \mathrm{ng} / \mathrm{ml}$ vs. $53.5 \pm 36.1 \mathrm{ng} / \mathrm{ml}, \mathrm{P}=0.072$ ), but did not affect zonulin levels at later measurements. Zonulin levels in serum and feces did not differ between normal-weighted and over-weighted subjects, or non-obese and obese subjects (data not shown), but were higher in men than in women $(93 \pm 30 \mathrm{ng} / \mathrm{ml} \mathrm{vs} .70 \pm 23 \mathrm{ng} / \mathrm{ml}$, $\mathrm{P}=0.035)$. 
Other inflammatory biomarkers. The other inflammatory biomarkers were unaffected during the dietary intervention. At follow-up, the levels of haptoglobin and thrombocytes were lowered compared with prior study start, whereas the values of albumin, CRP, and leukocytes were unaffected throughout the study (Table III).

Anthropometric, hormonal, and metabolic parameters. At inclusion, the mean weight was $89.8 \pm 15.0 \mathrm{~kg}$, BMI was $29.9 \pm 4.1 \mathrm{~kg} / \mathrm{m}^{2}$, and waist circumference was $107.3 \pm 10.9 \mathrm{~cm}$. Ninety percent of the patients $(n=27)$ were over-weighted whereas $50 \%(n=15)$ were obese. The weight was higher in men than in women $(97.5 \pm 11.6 \mathrm{~kg}$ vs. $83.9 \pm 14.9 \mathrm{~kg}$, $\mathrm{P}=0.011$ ), whereas the BMI did not differ between gender (29.5 \pm 2.4 vs. $\left.30.2 \pm 5.2 \mathrm{~kg} / \mathrm{m}^{2}, \mathrm{P}=0.592\right)$. During the intervention period of 12 weeks, the body weight was reduced by $6.2 \mathrm{~kg}$ ( $7 \%$ of total body weight) $(\mathrm{P}<0.001)$, accomplished by a reduction of $7.0 \mathrm{~cm}$ in waist circumference $(\mathrm{P}<0.001)$ and of $2.0 \mathrm{~kg} / \mathrm{m}^{2}$ in $\mathrm{BMI}(\mathrm{P}<0.001)$. These changes persisted to the same degree at follow-up 16 weeks later (data not shown).

Levels of glucose, HbA1c, C-peptide, and insulin were decreased during the dietary intervention, but only the HbA1c levels were still decreased at week 28 (Table IV). The insulin resistance calculated as HOMA2-IR was decreased throughout the study. Plasma levels of leptin was decreased at week 12 after dietary intervention $(\mathrm{P}<0.001)$, whereas resistin and visfatin levels did not decrease until week 28 (Table IV). Plasma levels of cholesterol and triglycerides were decreased during the study (data not shown).

Correlations between calprotectin, zonulin, and other inflammatory biomarkers and food components. There were no correlations between calprotectin and zonulin levels at baseline (data not shown). At week 12, there was a weak correlation between calprotectin in serum and zonulin in serum $\left(r_{s}=0.410\right.$, $\mathrm{P}=0.025)$ and calprotectin in feces and zonulin in feces $\left(r_{s}=0.326, P=0.079\right)$, as was also true at week $28\left(r_{s}=0.421\right.$, $\mathrm{P}=0.045$ vs. $\left.\mathrm{r}_{\mathrm{s}}=0.561, \mathrm{P}=0.005\right)$. There were no correlations between calprotectin and zonulin levels in serum and feces, respectively (Table V).

At baseline, no correlations were found between food composition of breakfast and calprotectin or zonulin in serum or feces (data not shown). At week 12, when all food except the breakfasts were standardized, energy percentage of protein in the breakfast was strongly correlated with calprotectin in serum $\left(r_{s}=0.499, P=0.008\right)$, and this correlation was further strengthened at follow-up (Table VI). Zonulin in serum had a tendency to correlate with energy percentage of protein and correlated inversely with energy percentage of carbohydrates (Table VI). No food components correlated with albumin, CRP, haptoglobin, leukocytes, or thrombocytes at any time point (data not shown).

Correlations between calprotectin and zonulin and anthropometry, endocrine profile, other inflammatory biomarkers and metabolic parameters. At baseline, serum calprotectin did not correlate with age $\left(r_{s}=0.006, P=0.975\right)$, weight $\left(r_{s}=-0.205\right.$, $\mathrm{P}=0.278), \mathrm{BMI}\left(\mathrm{r}_{\mathrm{s}}=0.018, \mathrm{P}=0.927\right)$, or waist circumference $\left(r_{s}=0.024, P=0.901\right)$. Accordingly, feces calprotectin did neither correlate with age $\left(r_{s}=-0.088, P=0.642\right)$, weight $\left(r_{s}=0.027\right.$,
$\mathrm{P}=0.889)$, $\mathrm{BMI}\left(\mathrm{r}_{\mathrm{s}}=0.204, \mathrm{P}=0.281\right)$, nor waist circumference $\left(r_{s}=0.114, \mathrm{P}=0.547\right)$.

On the contrary, serum zonulin did not correlate with age $\left(r_{s}=0.106, P=0.579\right)$, but correlated with weight $\left(r_{s}=0.453\right.$, $\mathrm{P}=0.012), \mathrm{BMI}\left(\mathrm{r}_{\mathrm{s}}=0.382, \mathrm{P}=0.037\right)$, and waist circumference $\left(r_{s}=0.432, P=0.017\right)$. Feces zonulin did correlate with age $\left(r_{s}=0.548, P=0.002\right)$ but not with weight $\left(r_{s}=-0.053, P=0.782\right)$, $\mathrm{BMI}\left(\mathrm{r}_{\mathrm{s}}=0.338, \mathrm{P}=0.068\right)$, or waist circumference $\left(\mathrm{r}_{\mathrm{s}}=0.087\right.$, $\mathrm{P}=0.648)$.

Calprotectin levels in serum correlated positively with leukocytes and resistin at baseline, and with CRP, leukocytes, LDL, PAI-1, resistin, and visfatin after dietary intervention. Calprotectin levels in feces correlated with leukocytes, glucose, and insulin at baseline (Table V). Serum zonulin correlated with GIP and glucagon, at baseline. After dietary intervention, it did not longer correlate with these hormones, but instead correlated with glucose, HbA1c, triglycerides, cholesterol, and LDL. Feces zonulin correlated with haptoglobin and insulin resistance at baseline, and HbAlc at follow-up. Both serum and feces zonulin were inversely correlated to cortisol (Table V). Levels of albumin, liver function tests, thrombocytes, and other hormones measured by Luminex and ELISA (CCK, ghrelin, GLP-1, leptin, and PYY) were not correlated with calprotectin or zonulin levels (data not shown).

\section{Discussion}

The main finding in this study was that calprotectin levels in serum tended to be elevated during the dietary intervention with an Okinawan-based Nordic diet, in correlation with energy percentage of protein and levels of CRP, leukocytes, LDL, PAI-1, resistin, visfatin, and zonulin. Feces calprotectin was elevated at baseline but decreased non-significantly throughout the study. Feces calprotectin vs. feces zonulin, and serum calprotectin vs. serum zonulin, did not correlate at baseline, but correlations were seen after the dietary intervention and at follow-up. Zonulin levels were elevated in serum at baseline, were increased in both serum and feces during the dietary intervention, and remained elevated in serum throughout the present study. At follow-up, zonulin levels in serum tended to correlate positively with energy percentage of protein and correlated inversely with energy percentage of carbohydrates. There were no correlations between serum and feces values of the two biomarkers. Clinically established methods to estimate acute inflammation were mainly unaffected, although haptoglobin and thrombocytes were lowered at follow-up.

Elevated calprotectin levels reflect an inflammatory state (4-6), and is used in clinical practice to follow the course of inflammatory bowel disease (IBD) (23). Elevated zonulin is suspected to reflect increased intestinal permeability and presence of chronic inflammation and disease $(12,14)$. One of the features of type 2 diabetes is a low-graded systemic inflammation (15), which may explain elevated levels of feces calprotectin and serum zonulin in our patients already at inclusion. Intake of non-steroidal anti-inflammatory drugs, malignancy, and gastrointestinal infections are confounding factors for elevated feces calprotectin (23). However, no of these factors were changed during the study period and no of the subjects suffered from IBD. 
Table IV. Hormonal and metabolic biomarkers.

\begin{tabular}{|c|c|c|c|c|c|c|c|}
\hline \multirow[b]{2}{*}{ Variable } & \multirow[b]{2}{*}{ Mean } & \multicolumn{2}{|c|}{$95 \%$ CI } & \multirow[b]{2}{*}{ Mean change } & \multicolumn{2}{|c|}{$95 \% \mathrm{CI}$} & \multirow[b]{2}{*}{ P-value } \\
\hline & & Lower & Upper & & Lower & Upper & \\
\hline \multicolumn{8}{|c|}{ Fasting Glucose (mmol/l) } \\
\hline Baseline & 9.71 & 8.54 & 10.87 & & & & \\
\hline Week 12 & 7.91 & 6.55 & 9.27 & -1.80 & -2.63 & -0.96 & $<0.001$ \\
\hline Week 28 & 9.28 & 7.71 & 10.85 & -0.42 & -1.58 & 0.73 & 0.466 \\
\hline \multicolumn{8}{|c|}{ HbA1c (mmol/mol) } \\
\hline Baseline & 61.57 & 56.42 & 66.72 & & & & \\
\hline Week 12 & 49.20 & 44.02 & 54.38 & -12.37 & -16.40 & -8.33 & $<0.001$ \\
\hline Week 28 & 54.36 & 48.83 & 59.90 & -7.20 & -11.68 & -2.72 & 0.002 \\
\hline \multicolumn{8}{|c|}{ C-peptide (nmol/l) } \\
\hline Baseline & 0.99 & 0.82 & 1.17 & & & & \\
\hline Week 12 & 0.88 & 0.70 & 1.05 & -0.11 & -0.21 & -1.02 & 0.022 \\
\hline Week 28 & 0.88 & 0.68 & 1.09 & -0.11 & -0.24 & 0.03 & 0.131 \\
\hline \multicolumn{8}{|c|}{ Insulin (mIU/l) } \\
\hline Baseline & 15.53 & 12.67 & 18.40 & & & & \\
\hline Week 12 & 11.67 & 8.80 & 14.53 & -3.87 & -6.43 & -1.30 & 0.004 \\
\hline Week 28 & 12.96 & 9.72 & 16.19 & -2.58 & -5.55 & 0.40 & 0.089 \\
\hline \multicolumn{8}{|c|}{ HOMA2-IR (U) } \\
\hline Baseline & 3.00 & 2.50 & 3.51 & & & & \\
\hline Week 12 & 2.37 & 1.82 & 2.91 & -0.64 & -0.98 & -0.30 & $<0.001$ \\
\hline Week 28 & 2.61 & 2.05 & 3.16 & -0.40 & -0.75 & -0.05 & 0.025 \\
\hline \multicolumn{8}{|c|}{ Cortisol (nmol/l) } \\
\hline Baseline & 367 & 337 & 397 & & & & \\
\hline Week 12 & 325 & 290 & 360 & -41 & -74 & -8 & 0.015 \\
\hline Week 28 & 348 & 315 & 382 & -18 & -50 & 13 & 0.249 \\
\hline \multicolumn{8}{|c|}{ PAI-1 (pg/ml) } \\
\hline Baseline & 72434 & 58869 & 85998 & & & & \\
\hline Week 12 & 66413 & 52402 & 80425 & -6020 & -12814 & 774 & 0.082 \\
\hline Week 28 & 71810 & 56705 & 86915 & -623 & -9455 & 8209 & 0.889 \\
\hline \multicolumn{8}{|c|}{ Resistin (pg/ml) } \\
\hline Baseline & 5234 & 3904 & 6565 & & & & \\
\hline Week 12 & 5043 & 3705 & 6382 & -191 & -1118 & 736 & 0.683 \\
\hline Week 28 & 4325 & 2915 & 5736 & -909 & -1938 & 119 & 0.082 \\
\hline \multicolumn{8}{|c|}{ Visfatin (pg/ml) } \\
\hline Baseline & 998 & 732 & 1264 & & & & \\
\hline Week 12 & 1115 & 816 & 1415 & 117 & -78 & 312 & 0.235 \\
\hline Week 28 & 812 & 535 & 1089 & -186 & -344 & -29 & 0.021 \\
\hline
\end{tabular}

The mean values and mean changes and 95\% confidence interval (CI) with lower and higher limits are presented for fasting levels of HbA1c (whole blood), Cortisol, C-peptide and insulin (serum), and glucose, leptin, plasminogen activator inhibitor-1 (PAI-1), resistin, and visfatin (plasma) at inclusion (baseline), 12 weeks after diet intervention, and 16 weeks after the end of diet intervention (week 28). Insulin resistance was measured by HOMA2-IR (22). Linear mixed model. P $\leq 0.05$ was considered statistically significant.

Weight reduction per se reduces systemic inflammation; the expression of pro-inflammatory factors are decreased and the expression of anti-inflammatory factors are increased (16). In addition, reduced energy intake; reduced hyperglycemia and hyperinsulinemia; a different food composition with lower GI/glycemic load and increased amount of fish, fibers, fruits and vegetables, and unsaturated fat; and healthy eating patterns reduce the chronic low-grade inflammation (16). Since the present study of Okinawan-based Nordic diet contained all components mentioned, and the diet evoked weight-reduction and improved metabolism, the elevated serum levels of zonulin and calprotectin during dietary intervention were therefore unexpected. In a previous report, zonulin levels in serum were elevated in obese subjects compared with normal-weighted 
Table V. Correlations between calprotectin, zonulin, and other biomarkers.

\begin{tabular}{|c|c|c|c|c|}
\hline & \multicolumn{2}{|c|}{ Baseline } & \multicolumn{2}{|c|}{ Week 12} \\
\hline & Correlation coefficient & P-value & Correlation coefficient & P-value \\
\hline \multicolumn{5}{|c|}{ Serum calprotectin } \\
\hline CRP & -0.108 & 0.571 & 0.459 & 0.011 \\
\hline Leukocytes & 0.368 & 0.049 & 0.475 & 0.008 \\
\hline Haptoglobin & 0.064 & 0.737 & 0.225 & 0.232 \\
\hline LDL & 0.080 & 0.672 & 0.377 & 0.040 \\
\hline PAI & 0.053 & 0.780 & 0.494 & 0.007 \\
\hline Resistin & 0.404 & 0.028 & 0.407 & 0.028 \\
\hline Visfatin & 0.297 & 0.111 & 0.529 & 0.005 \\
\hline \multicolumn{5}{|c|}{ Feces c+alprotectin } \\
\hline CRP & 0.296 & 0.113 & 0.267 & 0.154 \\
\hline Leukocytes & 0.387 & 0.038 & 0.187 & 0.321 \\
\hline Haptoglobin & 0.152 & 0.423 & 0.158 & 0.404 \\
\hline Glucose & 0.391 & 0.033 & 0.036 & 0.851 \\
\hline Insulin & 0.391 & 0.033 & 0.259 & 0.167 \\
\hline \multicolumn{5}{|l|}{ Serum zonulin } \\
\hline CRP & -0.026 & 0.892 & 0.194 & 0.305 \\
\hline Leukocytes & -0.095 & 0.623 & 0.130 & 0.494 \\
\hline Haptoglobin & -0.120 & 0.526 & -0.160 & 0.398 \\
\hline Glucose & 0.342 & 0.064 & 0.487 & 0.006 \\
\hline HbA1c & 0.254 & 0.175 & 0.369 & 0.045 \\
\hline TG & 0.046 & 0.808 & 0.416 & 0.022 \\
\hline Cholesterol & -0.041 & 0.831 & 0.400 & 0.029 \\
\hline LDL & -0.061 & 0.786 & 0.400 & 0.028 \\
\hline Cortisol & 0.038 & 0.842 & -0.429 & 0.020 \\
\hline GIP & 0.503 & 0.005 & 0.100 & 0.604 \\
\hline Glucagon & 0.445 & 0.014 & 0.115 & 0.553 \\
\hline \multicolumn{5}{|l|}{ Feces zonulin } \\
\hline CRP & 0.080 & 0.673 & -0.081 & 0.672 \\
\hline Leukocytes & 0.315 & 0.096 & 0.348 & 0.060 \\
\hline Haptoglobin & 0.367 & 0.046 & 0.160 & 0.398 \\
\hline $\mathrm{HbA1c}$ & 0.281 & 0.133 & 0.372 & 0.043 \\
\hline HOMA2-IR & 0.500 & 0.007 & 0.128 & 0.510 \\
\hline Cortisol & 0.302 & 0.104 & -0.369 & 0.049 \\
\hline
\end{tabular}

Insulin resistance was measured by HOMA2-IR (21). Correlation by Spearman's correlation test. $\mathrm{P} \leq 0.05$ was considered statistically significant. CRP, C-reactive protein; GIP, glucose-dependent insulinotropic polypeptide; PAI-1, plasminogen activator inhibitor-1.

subjects (25), and zonulin correlated with BMI, waist-to-hip ratio, plasma glucose levels, triglycerides, total cholesterol, insulin resistance, and systolic blood pressure $(26,27)$, in line with our correlations after dietary intervention. Our present findings of lower BMI, waist circumference, and insulin resistance, and lower levels of glucose, cholesterol, and triglycerides should have lowered the zonulin levels (28). Albeit all these changes, zonulin levels were increased after 12 weeks in both serum and feces and the increase in serum persisted at follow-up, when haptoglobin levels were decreased. This raises the hypothesis that the maturation of prehaptoglobin-2 to haptoglobin is stopped. The higher zonulin levels in men may depend on their higher weight. The tendency of lowered feces zonulin in those few with use of probiotics is in alignment with other studies (29).

Evaluation of a single breakfast in healthy volunteers with two different compositions showed that the Okinawan-based Nordic breakfast led to increased satiety and lower levels of glucose, C-peptide, insulin, and GIP (21), compared with a traditional control breakfast, whereas neither calprotectin nor zonulin levels were influenced (30). Although higher fat content, postprandial plasma levels of triglycerides were less increased after a single Okinawan-based breakfast, compared with the control breakfast with lower fat content (30). At baseline, there were no correlations between calprotectin and zonulin, neither in the present study in diabetes nor prior to the breakfast 
Table VI. Correlations of calprotectin and zonulin levels and breakfast components.

\begin{tabular}{|c|c|c|c|c|}
\hline & \multicolumn{2}{|c|}{ Correlation coeeficient } & \multicolumn{2}{|c|}{ P-value } \\
\hline & G & $\mathrm{E} \%$ & G & $\mathrm{E} \%$ \\
\hline \multicolumn{5}{|l|}{ Protein week 28} \\
\hline Serum Calprotectin & 0.365 & 0.687 & 0.687 & $<0.001$ \\
\hline Feces Calprotectin & 0.119 & -0.024 & 0.588 & 0.912 \\
\hline Serum Zonulin & -0.149 & 0.399 & 0.497 & 0.059 \\
\hline Feces Zonulin & 0.044 & 0.183 & 0.843 & 0.403 \\
\hline \multicolumn{5}{|l|}{ Carbohydrates week 28} \\
\hline Serum Calprotectin & -0.011 & -0.196 & 0.959 & 0.369 \\
\hline Feces Calprotectin & -0.075 & -0.063 & 0.733 & 0.774 \\
\hline Serum Zonulin & -0.229 & -0.422 & 0.293 & 0.045 \\
\hline Feces Zonulin & -0.045 & 0.116 & 0.837 & 0.599 \\
\hline \multicolumn{5}{|l|}{ Fat week 28} \\
\hline Serum Calprotectin & 0.011 & -0.186 & 0.959 & 0.397 \\
\hline Feces Calprotectin & 0.148 & -0.046 & 0.501 & 0.835 \\
\hline Serum Zonulin & -0.152 & 0.071 & 0.489 & 0.747 \\
\hline Feces Zonulin & -0.168 & -0.164 & 0.455 & 0.455 \\
\hline \multicolumn{5}{|l|}{ Fiber week 28} \\
\hline Serum Calprotectin & -0.224 & -0.356 & 0.304 & 0.096 \\
\hline Feces Calprotectin & -0.207 & -0.282 & 0.343 & 0.193 \\
\hline Serum Zonulin & -0.236 & -0.133 & 0.278 & 0.544 \\
\hline Feces Zonulin & -0.238 & -0.159 & 0.274 & 0.467 \\
\hline
\end{tabular}

Spearman's correlation test. $\mathrm{P} \leq 0.05$ was considered statistically significant.

intakes in healthy volunteers (30). However, a 12 week dietary intervention induced strong correlations between calprotectin and zonulin levels. The present changes in calprotectin and zonulin levels indicate that some food component in the Okinawan-based Nordic diet trigger an inflammatory response with increased calprotectin and zonulin release. Gliadin is the only food component so far described to induce zonulin release $(10,12)$. Since gliadin intake was reduced in the diet, another component must be responsible for this increase. The same breakfast was in the vast majority withheld during the follow-up, as was also parts of the composition of the main meals, reflected in the persistent elevation of zonulin levels.

Our diet had higher protein content than the traditional Nordic diet. When the effect of protein on the inflammation has been evaluated, mainly CRP and interleukins have been measured $(31,32)$. The effect on these biomarkers have been modest, and the protein content has therefore been estimated to be of minor importance in inflammation. There is no consensus of which laboratory measurement that best reflects the degree of chronic inflammation. We found that albumin, CRP, and leukocytes, the chemistry analyses used in clinical practice, are of no use to follow a chronic course after dietary intervention. Instead, haptoglobin and thrombocytes, which were altered during the study, are seldom used for this purpose. Only calprotectin and zonulin of the inflammatory biomarkers correlated with energy percentage of protein. This suggests that higher protein content in food may trigger an inflammation, reflected in certain biomarkers. This hypothesis is strengthened by that a protein-rich diet rendered higher fecal calprotectin in dogs, compared with a carbohydrate-rich diet (33). Chronic exposure of food content may be more important for calprotectin and zonulin secretion than a single stimulation, possibly due to inflammatory regulatory mechanisms (17,30).

Epidemiologic studies have shown that a diet with low GI or high fiber content are both associated with lower levels of CRP and TNF and insulin resistance (15). Nevertheless, contradictory results of whole-grain products are found in interventional studies (16). Subtle changes in the processing of whole grains may influence their inflammatory response. Furthermore, the health status of the study subjects may also determine the inflammatory response to specific food components (16). Wheat germ agglutinin (WGA) is the best studied cereal grain lectin. Unprocessed wheat germ, which are present in muesli, contains higher amounts of active WGA than processed wheat germ products (17). In vitro studies have shown that WGA stimulate the release of pro-inflammatory cytokines and activate the immune system (17). Human data on the influence of WGA on inflammatory biomarkers are missing. The introduction of higher fiber content and whole-grain products in the Okinawan-based Nordic diet processed to decrease glucose and lipid levels efficiently, may increase the secretion of inflammatory factors. The balance between lectins and polyphenols in the diet is important 
to regulate the pro-inflammatory and anti-inflammatory effects (34). A systemic review suggests more interventional studies to confirm the statement that the Modern Western diet is more inflammatory than other diets (35).

The present results underlie the importance of interventional studies. Associations in cross-sectional studies may reflect primary causality or secondary compensatory mechanisms, whereas prospective interventional studies describe a true sequence of events. When studying correlations, life style habits, medication, co-morbidity and so on may interfere with serum levels measured. Calculations must be adjusted for confounding factors, e.g., obese subjects have a greater food intake than lean subjects (25). The only intervention in our trial was dietary changes, and all patients received the same food, except breakfast. Although a dietary intervention with a suspected healthier diet is followed by weight reduction and improved glucose metabolism, these changes do not necessarily lead to reduced degree of inflammation. In parallel, increased fiber intake only has a moderate effect on maintenance of remission of IBD (36). Although some of the patients had an anamnesis of infection, surgery or bad compliance, this could not explain the inflammatory changes observed secondary to dietary intervention.

Weight reduction due to surgery or a pure reduction of energy intake led to lower levels of calprotectin in serum $(4,5)$, whereas we only found a non-significant decrease in feces and a tendency of increase in serum after a weight reduction due to a fat-rich and protein-rich dietary composition. In previous research, serum levels of calprotectin were elevated in obesity with or without diabetes compared to healthy subjects, and serum calprotectin levels correlated with BMI, waist circumference, insulin, and inflammatory markers $(4,5)$. The correlations with BMI and waist circumference could not be found in the present smaller study, where most patients were over-weighted or obese at start, which could affect the correlations. One study showed a correlation between calprotectin and glucose in serum (5), whereas most studies could not show such a correlation $(4,6)$, which suggest that calprotectin regulation is independent of glucose metabolism. Although calprotectin has been shown to be inversely correlated with HDL in many studies $(5,6)$, we could not confirm this finding.

A meta-analysis of the Mediterranean diet, which is similar to the Okinawan-based diet, described how this diet reduced concentrations of CRP and interleukins, with reduced insulin resistance and improved endothelial function (37). Endothelial dysfunction strictly correlates with insulin resistance and inflammation (37). The present elevation of feces calprotectin at baseline and its slight decrease at the same time as C-peptide, insulin, and insulin resistance were decreased, may reflect that hyperinsulinemia has a negative impact on the gut. This raises the hypothesis whether also epithelial function, and not only endothelial function, is related to inflammation and insulin resistance (37), and whether insulin resistance is a key factor for development of gastropathy in the same way as for nephropathy (38).

The strength of the study is that all analyses were performed with the same batch number and within 6 weeks after storage at $-80^{\circ} \mathrm{C}$, since zonulin is supposed to be degraded within 1-2 months in $-20^{\circ} \mathrm{C}$ (manufacturer's instruction). When comparing other studies in the field, most of them have not reported the storage of the samples, which may explain some of the differences between previous studies and the present study. The limitation of the present study is the absence of control group. However, a previous breakfast study showed that metabolic postprandial parameters differed between an ordinary meal and on Okinawan-based Nordic meal, in parallel with the present dietary intervention $(18,21)$. Further, a similar study has not shown any changes in metabolic parameters over the observational time period in a control group (39).

Adipokines, e.g., leptin, resistin, and visfatin, are hormones released from the adipose tissue with a central role in the control of energy metabolism, regulation of glucose and lipid metabolism, and insulin sensitivity (40). These hormones are supposed to be involved in the development of obesity, diabetes, inflammation, auto-immunity, and metabolic syndromes (41). In the present study, changes in calprotectin levels correlated with changes in resistin and visfatin levels, but not with leptin levels. This may depend on that resistin and visfatin are primarily secreted from abdominal fat tissue, whereas leptin is primarily secreted from subcutaneous fat tissue $(16,40,41)$. Furthermore, resistin and visfatin are also secreted from activated neutrophils (40), in parallel with calprotectin.

In conclusion, calprotectin and zonulin in serum and zonulin in feces are elevated after introduction of an Okinawan-based Nordic diet. The increase of serum levels of these biomarkers may depend on increased protein content of the food, since they correlate with energy percentage of protein. These findings deserve further experimental trials to identify pro-inflammatory components in the food.

\section{Acknowledgements}

We want to acknowledge Igelösa Life Science AB, Lund, whose staff supplied and prepared the breakfasts, and the staff at the Internal Medicine Research Group, Skåne University Hospital, Malmö, for performing the trials. The present study was supported by grants from Hans-Gabriel and Alice Trolle Wachtmeister's Foundation for Medical Research, King Gustaf $\mathrm{V}: \mathrm{s}$ and queen Victoria Free Maison's Foundation, Dir Albert Påhlsson's Foundation, Development Foundation of Region Skåne, and Foundation of Skåne University Hospital, Ernold Lundström Foundation.

\section{References}

1. Odink K, Cerletti N, Brüggen J, Clerc RG, Tarcsay L, Zwadlo G, Gerhards G, Schlegel R and Sorg C: Two calcium-binding proteins in infiltrate macrophages of rheumatoid arthritis. Nature 330: 80-82, 1987.

2. Zwadlo G, Brüggen J, Gerhards G, Schlegel R and Sorg C: Two calcium-binding proteins associated with specific stages of myeloid cell differentiation are expressed by subsets of macrophages in inflammatory tissues. Clin Exp Immunol 72: 510-515, 1988.

3. Ehrchen JM, Sunderkötter C, Foell D, Vogl T and Roth J: The endogenous Toll-like receptor 4 agonist S100A8/S100A9 (calprotectin) as innate amplifier of infection, autoimmunity, and cancer. J Leukoc Biol 86: 557-566, 2009.

4. Catalán V, Gómez-Ambrosi J, Rodríguez A, Ramírez B, Rotellar F, Valentí V, Silva C, Gil MJ, Fernández-Real JM, Salvador J and Frühbeck G: Increased levels of calprotectin in obesity are related to macrophage content: Impact on inflammation and effect of weight loss. Mol Med 17: 1157-1167, 2011. 
5. Ortega FJ, Sabater M, Moreno-Navarrete JM, Pueyo N, Botas $P$, Delgado E, Ricart W, Frühbeck G and Fernández-Real JM: Serum and urinary concentrations of calprotectin as markers of insulin resistance and type 2 diabetes. Eur J Endocrinol 167 $569-578,2012$

6. Pedersen L, Nybo M, Poulsen MK, Henriksen JE, Dahl J and Rasmussen LM: Plasma calprotectin and its association with cardiovascular disease manifestations, obesity and the metabolic syndrome in type 2 diabetes mellitus patients. BMC Cardiovase Disord 14: 196, 2014.

7. Eue I, Pietz B, Storck J, Klempt M and Sorg C: Transendothelial migration of $27 \mathrm{E} 10^{+}$human monocytes. Int Immunol 12 : 1593-1604, 2000.

8. Chiba H, Osanai M, Murata M, Kojima T and Sawada N: Transmembrane proteins of tight junctions. Biochim Biophys Acta 1778: 588-600, 2008.

9. Fasano A: Regulation of intercellular tight junctions by zonula occludens toxin and its eukaryotic analogue zonulin. Ann N Y Acad Sci 915: 214-222, 2000

10. Tripathi A, Lammers KM, Goldblum S, Shea-Donohue T, Netzel-Arnett S, Buzza MS, Antalis TM, Vogel SN, Zhao A Yang S, et al: Identification of human zonulin, a physiological modulator of tight junctions, as prehaptoglobin-2. Proc Natl Acad Sci USA 106: 16799-16804, 2009.

11. Sapone A, de Magistris L, Pietzak M, Clemente MG, Tripathi A Cucca F, Lampis R, Kryszak D, Cartenì M, Generoso M, et al: Zonulin upregulation is associated with increased gut permeability in subjects with type 1 diabetes and their relatives. Diabetes 55: 1443-1449, 2006

12. Fasano A: Intestinal permeability and its regulation by zonulin Diagnostic and therapeutic implications. Clin Gastroenterol Hepatol 10: 1096-1100, 2012

13. El Asmar R, Panigrahi P, Bamford P, Berti I, Not T, Coppa GV Catassi $\mathrm{C}$ and Fasano A: Host-dependent zonulin secretion causes the impairment of the small intestine barrier function after bacterial exposure. Gastroenterology 123: 1607-1615, 2002.

14. Vanuytsel T, Vermeire S and Cleynen I: The role of Haptoglobin and its related protein, Zonulin, in inflammatory bowel disease. Tissue Barriers 1: e27321, 2013.

15. Qi L and Hu FB: Dietary glycemic load, whole grains, and systemic inflammation in diabetes: The epidemiological evidence. Curr Opin Lipidol 18: 3-8, 2007.

16. Calder PC, Ahluwalia N, Brouns F, Buetler T, Clement K, Cunningham K, Esposito K, Jönsson LS, Kolb H,Lansink M, et al: Dietary factors and low-grade inflammation in relation to overweight and obesity. Br J Nutr 106 (Suppl 3): S5-S78, 2011.

17. de Punder K and Pruimboom L: The dietary intake of wheat and other cereal grains and their role in inflammation. Nutrients 5 : 771-787, 2013

18. Darwiche G, Höglund P, Roth B, Larsson E, Sjöberg T, Wohlfart B, Steen S and Ohlsson B: An Okinawan-based Nordic diet improves anthropometry, metabolic control, and health-related quality of life in Scandinavian patients with type 2 diabetes: A pilot trial. Food Nutr Res 60: 32594, 2016

19. Willcox DC, Scapagnini G and Willcox BJ: Healthy aging diets other than the Mediterranean: A focus on the Okinawan diet. Mech Ageing Dev 136-137: 148-162, 2014.

20. Norden: Nordic Nutrition Recommendations 2012, Integrating nutrition and physical activity. Nordic Council of Ministers. https://www.norden.org/en/theme/nordic-nutrition-recommendation/nordic-nutrition-recommendations-2012.

21. Ohlsson B, Höglund P, Roth B and Darwiche G: Modification of a traditional breakfast leads to increased satiety along with attenuated plasma increments of glucose, C-peptide, insulin, and glucose-dependent insulinotropic polypeptide in humans. Nutr Res 36: 359-368, 2016.

22. Diabetes Trials Unit, The Oxford Centre for Diabetes, Endocrinology and Metabolism: HOMA2 calculator, 2016. University of Oxford, Oxford, UK

23. Walsham NE and Sherwood RA: Fecal calprotectin in inflammatory bowel disease. Clin Exp Gastroenterol 9: 21-29, 2016.

24. World Health Organization: BMI classification. http://apps.who. int/bmi/index.jsp?introPage $=$ intro $3 . \mathrm{html}$.
25. Zak-Gołab A, Kocełak P, Aptekorz M, Zientara M, Juszczyk L, Martirosian G, Chudek J and Olszanecka-Glinianowicz M: Gut microbiota, microinflammation, metabolic profile, and zonulin concentration in obese and normal weight subjects. Int J Endocrinol 2013: 674106, 2013.

26. Zhang D, Zhang L, Zheng Y, Yue F, Russell RD and Zeng Y: Circulating zonulin levels in newly diagnosed Chinese type 2 diabetes patients. Diabetes Res Clin Pract 106: 312-318, 2014

27. Malyszko J, Koc-Zorawska E, Levin-Iaina N and Malyszko J: Zonulin, iron status, and anemia in kidney transplant recipients: Are they related? Transplant Proc 46: 2644-2646, 2014.

28. Kant P, Fazakerley R and Hull MA: Faecal calprotectin levels before and after weight loss in obese and overweight subjects. Int J Obes 37: 317-319, 2013.

29. Lamprecht M, Bogner S, Schippinger G, Steinbauer K, Fankhauser F, Hallstroem S, Schuetz B and Greilberger JF: Probiotic supplementation affects markers of intestinal barrier, oxidation, and inflammation in trained men; a randomized, double-blinded, placebo-controlled trial. J Int Soc Sports Nutr 9: $45,2012$.

30. Ohlsson B, Darwiche G, Roth B and Höglund P: Two meals with different carbohydrate, fat and protein contents render equivalent postprandial plasma levels of calprotectin, cortisol, triglycerides and zonulin. Int J Food Sci Nutr 67: 872-880, 2016

31. Zhou LM, Xu JY, Rao CP, Han S, Wan Z and Qin LQ: Effect of whey supplementation on circulating $\mathrm{C}$-reactive protein: A meta-analysis of randomized controlled trials. Nutrients 7 : 1131-1143, 2015.

32. Ticinesi A, Meschi T, Lauretani F, Felis G, Franchi F, Pedrolli C, Barichella M, Benati G, Di Nuzzo S, Ceda GP and Maggio M: Nutrition and Inflammation in older individuals: Focus on Vitamin D, n-3 polyunsaturated fatty acids and whey proteins. Nutrients 8: 186, 2016.

33. Hang I, Heilmann RM, Grützner N, Suchodolski JS, Steiner JM, Atroshi F, Sankari S, Kettunen A, de Vos WM, Zentek J and Spillmann T: Impact of diets with a high content of greaves-meal protein or carbohydrates on faecal characteristics, volatile fatty acids and faecal calprotectin concentrations in healthy dogs. BMC Vet Res 9: 201, 2013.

34. Fardet A: New hypotheses for the health-protective mechanisms of whole-grain cereals: What is beyond fibre? Nutr Res Rev 23: 65-134, 2010.

35. Schwab U, Lauritzen L, Tholstrup T, Haldorssoni T, Riserus U, Uusitupa $M$ and Becker W: Effect of the amount and type of dietary fat on cardiometabolic risk factors and risk of developing type 2 diabetes, cardiovascular diseases, and cancer: A systematic review. Food Nutr Res 58: 25145, 2014.

36. Wedlake L, Slack N, Andreyev HJ and Whelan K: Fiber in the treatment and maintenance of inflammatory bowel disease: A systematic review of randomized controlled trials. Inflamm Bowel Dis 20: 576-586, 2014

37. Casas R, Sacanella E and Estruch R: The immune protective effect of the Mediterranean diet against chronic low-grade inflammatory diseases. Endocr Metab Immune Disord Drug Targets 14: 245-254, 2014

38. Mima A: Inflammation and oxidative stress in diabetic nephropathy: New insights on its inhibition as new therapeutic targets. J Diabetes Res 2013: 248563, 2013.

39. Adamsson V, Reumark A, Fredriksson IB, Hammarström E, Vessby B, Johansson G and Risérus U: Effects of a healthy Nordic diet on cardiovascular risk factors in hypercholesterolaemic subjects: A randomized controlled trial (NORDIET). J Intern Med 269: 150-159, 2011.

40. Al-Suhaimi EA and Shehzad A: Leptin, resistin and visfatin: The missing link between endocrine metabolic disorders and immunity. Eur J Med Res 18: 12, 2013.

41. Garten A, Schuster S, Penke M, Gorski T, de Giorgis T and Kiess W: Physiological and pathophysiological roles of NAMPT and NAD metabolism. Nat Rev Endocrinol 11: 535-546, 2015. 